\title{
Reflections on the impact of "flatten the curve" on interdependent workforce sectors
}

\author{
Joost Santos ${ }^{1}$ (D) \\ Published online: 14 May 2020 \\ ○) Springer Science+Business Media, LLC, part of Springer Nature 2020
}

\begin{abstract}
The expression "flatten the curve" has gained significant attention in the midst of the COVID-19 pandemic. The idea is to decrease and/or delay the peak of an epidemic wave so as not to strain or exceed the capacity of healthcare systems. There has been an increasing number of policy recommendations across the globe that favor the use of nonpharmaceutical interventions (NPIs) to flatten the curve. NPIs encompass containment, suppression, and mitigation measures such as quarantine, travel restrictions, and business closures. This paper provides perspectives on the impact of containment, suppression, and mitigation measures on interdependent workforce sectors. Reflections on the trade-offs between flattening the curve versus personal liberty and socioeconomic disparities are also presented in this paper.
\end{abstract}

Keywords COVID-19 $\cdot$ Coronavirus $\cdot$ Pandemic $\cdot$ Input-output model $\cdot$ Disaster risk management $\cdot$ Workforce

\section{Evolution of the "flatten the curve"}

The COVID-19 pandemic has caused unprecedented impacts on the global society, threatening to exhaust the capacity of health care systems and compromising the operations of many economic sectors. The "flatten the curve" graph, whose origin can be traced back to the Centers for Disease Control and Prevention (CDC 2007), has become a popular visualization tool in the midst of COVID-19 pandemic to explain the benefits of containment, suppression, and mitigation measures. Since CDC developed the graph, several versions were created such as the use of different color shadings to contrast the epidemic waves associated with different intervention scenarios (see for example, London and Milan 2020). Furthermore, in a Twitter post by Harris (2020), a horizontal line was added to represent the healthcare system capacity, which was featured in a New York Times article (Roberts 2020). Animation has also been created to illustrate ways with which the curve can be flattened (Wiles 2020; Stevens 2020).

Joost Santos

joost@gwu.edu

1 George Washington University, Washington, DC, USA

\section{Pandemic intervention measures}

Recently, the WEIGHT framework (Santos et al. 2020) was conceptualized to explore the multiple dimensions of disasters. The WEIGHT is an acronym that partitions disaster risk management into systems (Workforce, Economy, Infrastructure) and context (Geography, Hierarchy, Time). Nonetheless, the specific focus of the perspective shall be on the analysis of the economic losses that may arise from pandemic-related workforce absenteeism. Workforce recovery analysis in the context of disaster preparedness deserves an equitable level of importance and emphasis placed on critical infrastructure systems. Disaster management often tends to focus more on restoring damaged infrastructure systems to their pre-disaster levels, consequently leaving relatively less attention on managing losses that could stem from workforce disruptions and related factors. The analysis of workforce recovery relative to critical infrastructure protection still remains underdeveloped. In contrast to the numerous publications on infrastructure systems in the context of disaster risk management, a disproportionately low number of disaster-related research articles focus on the workforce. ${ }^{1}$

\footnotetext{
${ }^{1}$ For example, Web of Science reported 86,365 articles containing the word disaster. When disaster was combined with the word infrastructure, 5688 articles remained. In contrast, 291 articles remained when disaster was combined with workforce. Search was conducted on April 3, 2020
} 
Effective pharmaceutical interventions (e.g., vaccines, antivirals) are not immediately available for novel viruses, like the coronavirus associated with COVID-19. Hence, health agencies across the globe rely on nonpharmaceutical interventions, or NPIs, to manage the spread of infections. Examples of NPIs include hand hygiene, personal protective equipment, social distancing, travel restrictions, and school/ work closures, among others. Several recent articles have distinguished several categories of NPIs: containment, suppression, and mitigation.

Containment is the approach of managing individual infection chains (e.g., broad testing, contact tracing, isolation of travelers coming from high-prevalence areas, travel restrictions, among others). Containment is effective only when applied prior to the occurrence of community transmission, or after community transmission has been adequately suppressed. On the other hand, suppression aims to decrease the basic reproduction number (denoted by R0 in epidemiology literature) to a value lower than 1 and bring community transmission down to levels where implementation of containment will be feasible. Suppression measures are more aggressive and are typically applied at the early onset of the outbreak to keep infections to a minimum (Pueyo 2020). Suppression measure include mandated quarantine $^{2}$ in addition to implementing some of the containment measures like broad testing and travel restrictions). Furthermore, enforcement of quarantine would consequently lead to suspension of business and school operations, as well as large gatherings, during the pandemic period. Although both containment and suppression measures can be effective in decreasing the duration of the first wave, the likelihood and severity of a second or subsequent waves can be more extreme in the absence of vaccines, relative to mitigation. Finally, mitigation ("flatten the curve") does not aim to completely stop the transmission, but to lower R0 enough sufficiently that the medical system can handle peak demand. Mitigation measures aim to delay further spread of the disease such as social distancing, personal protective equipment (PPE), and hand hygiene. The advantages are that it requires less extreme restriction on contact, and that at the end of mitigation phase enough people would have contracted the virus, developed herd immunity, and are at a low or negligible risk of reinfection.

Several articles have discussed the distinctions in containment, suppression and mitigation measures in terms of their benefits as well as their implementation challenges (see, for examples, Ferguson et al. 2020). Regardless of whether the implementation of such measures is mandated or advisory, workforce-related disruption is an inevitable outcome of

\footnotetext{
2 Some countries have used terms such as lockdown, stay at home, or enhanced community quarantine.
}

disease outbreaks. A vast majority of the previous literature focused on workforce absenteeism due to personal illness or provision of care to sick family members. Nonetheless, the phenomenon of "forced" workforce absenteeism is unprecedented and is an area that has gained attention due to the COVID-19 pandemic. Forced workforce absenteeism is a consequence of regulations such as quarantine, travel bans/ restrictions, and mandated business closures.

\section{Epi curves, economic input-output data, and workforce disruptions}

Health agencies publish data on the number of infections on affected regions, which when normalized will create a time series of attack rates as the pandemic progresses from the first documented case to the peak, and until acceptable recovery is reached. This is typically referred to as epidemic curve (or epi curve). An epi curve can be constructed from data published by health agencies and research institutions. ${ }^{3}$ Typically, the y-axis of an epi curve shows the number of people infected by the disease, while the $\mathrm{x}$-axis depicts the time in days. The daily number of new infections can be expressed in terms of a percentage relative to the population of the affected region. This percentage is typically referred to as the attack rate 4 in epidemiological literature. Using the attack rate data in epi curves, it is possible to estimate the resulting disruption to various workforce sectors.

When a production factor is degraded (e.g., workforce disruptions during a pandemic), the output of a dependent economic sector can be estimated. This can be done by computing the ratio of the contribution of workforce relative to the total production output of each sector. Economic sectors and infrastructure systems depend on their workforce in varying degrees. As the level of labor dependence increases for a sector, its expected economic loss is also expected to rise in the event of workforce-debilitating events, like pandemics. Some sectors are less labor-intensive than others. A case in point, automation in sectors such as advanced manufacturing has reduced dependence on workforce, which were traditionally labor-intensive. Furthermore, it is possible to account for the flexibility of some economic sectors to perform workforce continuity strategies (e.g., teleworking), in which healthy workers in quarantine are assumed to be able

\footnotetext{
${ }^{3}$ See for example, the regularly updated coronavirus resource center developed by Johns Hopkins University and Medicine, https://coron avirus.jhu.edu/map.html.

4 The CDC website uses a more formal term "incidence proportion" in lieu of attack rate. See https://www.cdc.gov/csels/dsepd/ss1978/ lesson3/section2.html.
} 
to work remotely; hence reducing the impact of business closures.

Using economic input-output data from national statistical agencies, ${ }^{5}$ the efficacy of containment, suppression, and mitigation measures can be compared against a baseline epi curve, where minimal intervention is imposed. Simulation results indicate that strict suppression measures can significantly flatten the curve (James et al. 2020). In addition to relieving the pressure off the healthcare system, such measures can also decrease the expected economic loss relative to the baseline (Orsi and Santos 2010). This result may be counterintuitive at first since suppression measures (e.g., quarantine, travel restrictions, and business closures, among others) arguably generate costly opportunity losses. Nonetheless, the baseline scenario-which assumes significantly fewer closures-may lead to steeper and faster incidences of absenteeism due to more workers getting sick, caring for sick family members, or worse, dying — ultimately creating a surge in economic losses. Consistently, findings from the research work of Sergio Correia et al. (2020) concluded that US cities that implemented more aggressive and prolonged social distancing measures emerged with higher employment growth rates several years after the 1918 Spanish Flu pandemic. Nonetheless, an important caveat has to be made with the complexities and uncertainties surrounding COVID-19. To wit, steep escalation of unemployment rates and sector-specific losses are currently being experienced worldwide and there will come a point in time that prolonged implementation of suppression and mitigation measures may result in global economic collapse. Hence, government assistance and creative approaches ${ }^{6}$ have to be put in place to cushion the losses to vulnerable sectors such as restaurants, retail stores, personal care services, and entertainment, among others.

Recent articles cautioned that with minimal intervention, COVID-19 will be responsible for mortalities in the magnitude of hundreds of thousands, if not, millions (Pueyo 2020; Yong 2020). Hence, expedited production of PPEs, mass testing, enforced social distancing, and clear national/global coordination policies are needed to decrease the magnitude of such dire consequences to a minimum. A case in point, $\mathrm{Yu}$ and Aviso (2020) recently proposed a conceptual multiscale hierarchical framework to better coordinate supply chains at the firm, national, and global levels in the aftermath of disease outbreaks.

\footnotetext{
${ }^{5}$ For example, the US Bureau of Economic Analysis publishes IO data for varying levels of sector aggregation. See https://www.bea. gov/industry/input-output-accounts-data.

${ }^{6}$ Examples include curbside pick-up or delivery for stores and restaurants. Also, sectors that have leveraged information technology and virtual services have also seen an unprecedented surge in their demand.
}

Why then are governments cautious and generally averse with the implementation of containment, suppression, and mitigation measures? The main argument is that such measures may impinge on personal liberty. An extended lockdown, for example, will be difficult to accept by most. Previous studies (see, for example, Hawkley and Capitanio 2015) have also concluded that social isolation can adversely affect mental health, and can increase the risk of anxiety, depression, and substance use. Alafrangy (2020), who worked in NASA's Human Exploration Research Analog (HERA) project, relayed his first-hand experience living in a confined environment for 45 days:

The impact of isolation on humans, which are by nature social beings, can be profound. My time in HERA allowed me to develop a few techniques to mitigate the effects of isolation, which can be applicable to the current COVID-19 situation. I learned that following a strict schedule helped me keep busy and remain active. As the days and weeks went by, I noticed my progress with various tasks and experiments, which undoubtedly fueled me with energy and focus to keep going. Conflict within confinement is bound to arise as nobody is immune to it. It is not difficult, however, to understand that this isolation affects everyone, and that people may react differently.

Indeed, personal liberty needs to be carefully balanced against the uncertain consequences that may emerge from a pandemic, especially if it is allowed to run its course with minimal intervention. However, containment, suppression, and mitigation measures are not a panacea. They may work effectively in the current wave but will not guarantee the avoidance of future waves. Hence, vaccines, antivirals, and other pharmaceutical interventions need to be developed at the quickest time possible to thwart the potential resurgence of the virus. Furthermore, government officials and policymakers also need to be mindful of other negative side effects of pandemic interventions. To wit, enforcement of quarantine may further expose and amplify the inequality across various socioeconomic segments of the population. The impact of COVID-19 on lower income groups has been found to be much more profound, further impeding their ability to access basic resources, employment, as well as services such as healthcare and online education, among others. Studies also have indicated that low-income groups are more susceptible to contracting the disease itself, which further compounds the socioeconomic disparities associated with COVID-19.

In sum, containment, suppression, and mitigation measures can have a significant impact on the extent to which the curve can be flattened; consequently reducing the impact on the workforce, healthcare systems, and continuity of government, among others. The COVID-19 pandemic is an 
unprecedented disaster that has exposed major challenges and constraints in our socioeconomic and infrastructure systems. Indeed, current disaster preparedness and resilience practices need to be reviewed, re-evaluated, and significantly enhanced to minimize the impact of pandemics and other future disasters on all facets of our global society.

Funding Funding was provided by National Science Foundation (Grant No. 1832635).

\section{Compilance with ethical standards}

Conflict of interest The author has no conflict of interest for this article.

\section{References}

Alafrangy M (2020) Re: Question on NASA's human exploration research analog (HERA). Message to Joost Santos. 5 April 2020

Centers for Disease Control and Prevention (2007) Interim pre-pandemic planning guidance: community strategy for pandemic influenza mitigation in the United States: early, targeted, layered use of nonpharmaceutical interventions. https://stacks.cdc.gov/view/ cdc/11425. Accessed 7 April 2020

Correia S, Luck S, Verner E (2020) Pandemics depress the economy, public health interventions do not: evidence from the 1918 Flu. https://papers.ssrn.com/sol3/papers.cfm?abstract_id=3561560. Accessed 7 April 2020

Ferguson NM, Laydon D, Nedjati-Gilani G, Imai N, Ainslie K, Baguelin M, Bhatia S, Boonyasiri A, Cucunubá Z, Cuomo-Dannenburg G, Dighe A, Dorigatti I, Fu H, Gaythorpe K, Green W, Hamlet A, Hinsley W, Okell LC, van Elsland S, Thompson H, Verity R, Volz E, Wang H, Wang Y, Walker PGT, Walters C, Winskill P, Whittaker C, Donnelly CA, Riley S, Ghani AC (2020). Impact of non-pharmaceutical interventions (NPIs) to reduce COVID-19 mortality and healthcare demand. COVID-19 Reports, Faculty of Medicine, Imperial College, London. doi: 10.25561/77482.

Harris D (2020) Important to remember that \#Covid-19 epidemic control measures may only delay cases, not prevent. However, this helps limit surge and gives hospitals time to prepare and manage.
It's the difference between finding an ICU bed \& ventilator or being treated in the parking lot tent. https://twitter.com/drewaharri s/status/1233267475036372992. Accessed 7 April 2020

Hawkley LC, Capitanio JP (2015) Perceived social isolation, evolutionary fitness and health outcomes: a lifespan approach. Philos Trans R Soc B 370(1669):20140114. https://doi.org/10.1098/ rstb.2014.0114

James A, Hendy SC, Plank MJ, Steyn N (2020). Suppression and mitigation strategies for control of COVID-19 in New Zealand. https ://cpb-ap-se2.wpmucdn.com/blogs.auckland.ac.nz/dist/d/75/files /2017/01/Supression-and-Mitigation-Strategies-New-ZealandTPM-1.pdf. Accessed 7 April 2020

London and Milan (2020) Covid-19 is now in 50 countries, and things will get worse. The Economist. https://www.economist.com/brief ing/2020/02/29/covid-19-is-now-in-50-countries-and-things-willget-worse. Accessed 7 April 2020

Orsi MJ, Santos JR (2010) Probabilistic modeling of workforce-based disruptions and input-output analysis of interdependent ripple effects. Econ Syst Res 22(1):3-18

Pueyo T (2020) Coronavirus: the hammer and the dance, what the next 18 months can look like, if leaders buy us time. https://mediu m.com/@ tomaspueyo/coronavirus-the-hammer-and-the-dance -be9337092b56. Accessed 7 April 2020

Roberts S (2020) Flattening the coronavirus curve. https://www.nytim es.com/article/flatten-curve-coronavirus.html. Accessed 7 April 2020

Santos J, Yip C, Thekdi S, Pagsuyoin S (2020) Workforce/population, economy, infrastructure, geography, hierarchy, and time (WEIGHT): reflections on the plural dimensions of disaster resilience. Risk Anal 40(1):43-67

Stevens H (2020) Why outbreaks like coronavirus spread exponentially, and how to "flatten the curve." https://www.washingtonpost.com/ graphics/2020/world/corona-simulator/?fbclid=IwAR0KWReI 9vtrX0BvzEssTdr3GuhEuytyt42HPrF8BjOJGiVNdc0s9rFen4 U\&utm_source=reddit.com. Accessed 7 April 2020

Wiles S (2020) The three phases of COVID-19 and how we can make it manageable. https://twitter.com/SiouxsieW/status/1237275231 783284736. Accessed 7 April 2020

Yong E (2020). How the pandemic will end. The Atlantic. https:// www.theatlantic.com/health/archive/2020/03/how-will-coronaviru s-end/608719/. Accessed 7 April 2020

Yu K, Aviso KB (2020) Modelling the economic impact and ripple effects of disease outbreaks. Process Integr Optim Sustain. https ://doi.org/10.1007/s41660-020-00113-y 